\title{
Neutrino oscillations in a quantum processor
}

\author{
C. A. Argüelles ${ }^{1}$ and B. J. P. Jones $\oplus^{2}$ \\ ${ }^{1}$ Laboratory for Nuclear Science, Department of Physics, Massachusetts Institute of Technology, Cambridge, Massachusetts 02139, USA \\ ${ }^{2}$ Department of Physics, University of Texas at Arlington, Arlington, Texas 76019, USA
}

(Received 19 July 2019; published 13 December 2019)

\begin{abstract}
Quantum computing technologies promise to revolutionize calculations in many areas of physics, chemistry, and data science. Their power is expected to be especially pronounced for problems where direct analogs of a quantum system under study can be encoded coherently within a quantum computer. A first step toward harnessing this power is to express the building blocks of known physical systems within the language of quantum gates and circuits. In this paper we present a quantum calculation of an archetypal quantum system: neutrino oscillations. We define gate arrangements that implement the neutral lepton mixing operation and neutrino time evolution in two-, three-, and four-flavor systems. We then calculate oscillation probabilities by coherently preparing quantum states within the processor, time evolving them unitarily, and performing measurements in the flavor basis, with close analogy to the physical processes realized in neutrino oscillation experiments. Evaluations on publicly available quantum processors obtain excellent agreement with classical calculations. We provide recipes for modeling oscillation in the standard three-flavor paradigm as well as beyond-standard-model scenarios, including systems with sterile neutrinos, nonstandard interactions, Lorentz symmetry violation, and anomalous decoherence.
\end{abstract}

DOI: 10.1103/PhysRevResearch.1.033176

\section{INTRODUCTION}

The unexpected and Nobel Prize-winning discovery of neutrino oscillations [1] has led to a program of experiment and theory that has shaped the understanding of the role of neutrinos in the Universe. The spontaneous transition of neutrino flavor over macroscopic distances, a phenomenon known as neutrino oscillations due to its periodic behavior, demonstrates that neutrinos have masses that are nonzero but uniquely small. This smallness suggests connections to highscale physics [2-4] and may be related directly to the predominance of matter over antimatter abundances in the Universe [5]. Studies of neutrino oscillations have thus contributed and will continue to contribute greatly to our understanding of nature.

Experiments measure neutrino oscillations by studying a neutrino beam's flavor composition at different energies $E$ and baselines $L$ [6]. Oscillation refers to spontaneous transformation between the three neutrino flavors $v_{e}, v_{\mu}$, and $v_{\tau}$ during flight. This is due to dephasing of the neutrino wave functions during propagation arising from a misalignment between the flavor and mass bases. In the absence of strong matter interactions, and when two neutrino mass states dominate the oscillation, a sinusoidal flavor variation as a function of $L / E$ is characteristic. For oscillations in matter $[7,8]$ and with three neutrinos participating, more complex functional forms are

Published by the American Physical Society under the terms of the Creative Commons Attribution 4.0 International license. Further distribution of this work must maintain attribution to the author(s) and the published article's title, journal citation, and DOI. observed [9]. Well-studied neutrino sources, in which neutrino flavor changing has been observed, include $v_{\mu}$ and $\bar{v}_{\mu}$ production by decays of charged pions from accelerators $[10,11]$ or in cosmic-ray air showers $[12,13]$, production of $\bar{v}_{e}$ by fission in nuclear reactors $[14,15]$, and production of $v_{e}$ by nuclear fusion in the Sun [16]. Neutrino oscillations have been shown to violate the Leggett-Garg inequality [17], a time domain version of Bell's classic argument [18], which illustrates that they are a truly quantum mechanical phenomenon with no possible description in terms of hidden classical variables.

For neutrino oscillations to be observable, quantum coherence between the neutrino mass basis states must be maintained over the flight distance of the neutrino [19], which in some experiments is thousands of kilometers. Neutrinos are thus very-long-baseline quantum interferometers and they have been used as such to perform fundamental tests of quantum mechanics [20,21] and Lorentz invariance [22-24], in order to search for evidence of quantum gravity $[25,26]$ and violations of the equivalence principle [27]. The expected decoherence of oscillating neutrinos via wave-packet separation has been studied theoretically [28], but not yet observed in experiments.

Although quantum computing has been predominantly associated with extensive database queries [29] or number factorization [30], it was realized early in its conceptual development that a natural connection between simulation of quantum systems and quantum computers exists [31] (see [32] for a recent review on quantum simulation). An advantage of quantum computers over classical systems is the ability to perform actual Hamiltonian evolution rather than emulate it. In particle physics we often deal with high-particle multiplicity processes; for example, in high-energy collider experiments quantum computers have been noted to be advantageous in the 
simulation [33] and reconstruction [34] of hadronic showers. Other examples of physics frontiers that may be substantially advanced by quantum computation include modeling in nuclear physics [35], many-body effects in condensed matter systems [36], and quantum chromodynamics [37], among others.

This work demonstrates the processing of three-neutrino flavor information in a quantum simulation, executing an analogous Hamiltonian evolution to generate neutrino flavor oscillations. Such encoding and evolution is a vital building block on which more advanced quantum simulations involving neutrino flavor can be constructed. Systems that could particularly benefit from a quantum algorithmic approach to neutrino flavor evolution include those where collective neutrino oscillations $[38,39]$ are relevant, such as in supernovae or the early Universe [40]. In these cases the quantum Boltzmann equations that yield the evolution of the neutrino population can only be solved approximately [41] or by specialized numerical techniques $[42,43]$. This work demonstrating encoding of neutrino flavor structure and oscillation represents an important step toward addressing such problems using quantum processors.

Recently, publicly accessible quantum processors were made available online as part of the IBM Q project and these can be used for novel research into quantum processing [44]. Although the technology remains imperfect, with error rates per gate operation of $O(0.1 \%)$ and per qubit read of $O(5 \%)$ prohibiting very lengthy calculations, the platform provides a test bed for exploring quantum solutions to computational problems and finding ways to reexpress calculations in the language of quantum circuits.

In this paper, we present a quantum simulation of neutrino flavor oscillations. After illustrating how to encode the twoneutrino system evolution in a quantum computer with a single qubit, we proceed to implement the less intuitive threeneutrino system realized on a subspace of a two-qubit Hilbert space. The primary challenges involved are the implementation of the Pontecorvo-Maki-Nakagawa-Sakata (PMNS) [6] operation, which relates the flavor and mass neutrino eigenstates, and the time-evolution operator of the system in the computational basis. After testing that our quantum circuit reproduces the quantum neutrino oscillation probability on the IBM Q public quantum computer, we conclude with a brief discussion of how to include more complex phenomena including sterile neutrinos, matter effects, nonstandard interactions, Lorentz symmetry violation, and decoherence within the quantum algorithm.

\section{TWO-FLAVOR NEUTRINO OSCILLATION}

Two-flavor neutrino oscillations involve a Hilbert space of two dimensions. This can be represented on a single qubit, via the basis choice

$$
|0\rangle=\left|v_{1}\right\rangle=\left(\begin{array}{l}
1 \\
0
\end{array}\right), \quad|1\rangle=\left|v_{2}\right\rangle=\left(\begin{array}{l}
0 \\
1
\end{array}\right) .
$$

The rotation into the flavor basis requires a unitary operation via the two-dimensional PMNS matrix. The reduced PMNS operation is defined such that $\left|v_{e}\right\rangle=U_{\mathrm{PMNS}}^{2 \times 2 \dagger}|0\rangle$ and $\left|v_{\mu}\right\rangle=$ $U_{\mathrm{PMNS}}^{2 \times 2 \dagger}|1\rangle$. The most general unitary transformation applicable to a single-qubit system, which must be able to support the $2 \times 2$ PMNS operation, is encoded in the IBM quantum computer by the three-parameter $U 3$ gate

$$
U 3(\Theta, \phi, \lambda)=\left(\begin{array}{cc}
\cos \frac{\Theta}{2} & -\sin \frac{\Theta}{2} e^{i \lambda} \\
\sin \frac{\Theta}{2} e^{i \phi} & \cos \frac{\Theta}{2} e^{i(\lambda+\phi)}
\end{array}\right) .
$$

For the two-neutrino system, oscillation probabilities depend only on one of the parameters of $U 3$, for the following reasons [9].

(i) The parameter $\phi$ can be removed by a redefinition of the $\left|v_{\mu}\right\rangle$ basis state via $\left|v_{\mu}\right\rangle \rightarrow e^{-i \phi}\left|v_{\mu}\right\rangle$. This corresponds to rephasing the charged muon field, under which the standard model Lagrangian is invariant. Without loss of generality, we can set $\phi=0$.

(ii) The parameter $\lambda$ could similarly be removed by rephasing the $\left|\nu_{2}\right\rangle$ field $\left|\nu_{2}\right\rangle \rightarrow e^{i \lambda}\left|\nu_{2}\right\rangle$. The Lagrangian is only invariant under this redefinition if the neutrinos are Dirac particles. If they are Majorana particles, on the other hand, this phase is physical and must be maintained in the Lagrangian. However, it can be shown the Majorana phase $\lambda$ does not influence neutrino oscillations [45] and general oscillation probabilities can be calculated under the assumption $\lambda=0$.

We thus connect to the conventionally defined $2 \times 2$ neutrino PMNS matrix via the definition

$$
U_{\mathrm{PMNS}}^{2 \times 2}=U 3(2 \theta, 0,0)=\left(\begin{array}{cc}
\cos \theta & -\sin \theta \\
\sin \theta & \cos \theta
\end{array}\right) .
$$

The IBM Q U3 gate used in this way has a simple interpretation, as a rotation around the $Y$ axis in the Pauli representation. To prepare a neutrino flavor state, we can apply the PMNS operation either to the $|0\rangle$ state to prepare $\left|v_{e}\right\rangle$ or to the $|1\rangle$ state to prepare $\left|v_{\mu}\right\rangle$. The input qubits in a quantum computation conventionally initialize to $|0\rangle$, and the |1) state can be prepared by application of the Pauli- $X$ gate, $|1\rangle=X|0\rangle$. The preparation of electron and muon neutrino flavor states, as well as $m_{1}$ and $m_{2}$ mass states in the two-flavor basis, is shown in terms of quantum circuit elements in Fig. 1.

Oscillation probabilities can be calculated by time evolving the initial flavor state vector in with the appropriate timeevolution operator $\mathcal{U}$ and then measuring in the flavor basis. Only relative phases between mass states are relevant for oscillations, and so without loss of generality we can measure all phases relative to the $m_{1}$ basis state. The time-evolution operation can thus be encoded in an $S$ gate

$$
\mathcal{U}(t)=S(\phi)=\left(\begin{array}{cc}
1 & 0 \\
0 & e^{i \phi}
\end{array}\right),
$$

where $\phi=\Delta m^{2} t / 2 E \hbar$. In the two-flavor system, we thus find a particularly simple representation of the PMNS and time-evolution gates, shown in Figs. 1(b) and 1(c). Examples of circuits that realize various two-flavor oscillation scenarios are given in Fig. 2. With the quantum circuit defined, we can proceed to evaluate oscillation probabilities on the quantum processor. We run 1024 trials and count flavor measurement outcomes to establish oscillation probabilities in the twoflavor system. Figure 3 shows the comparison of the quantum calculation to the theoretical expectation for parameters relevant to the $v_{e}$ disappearance in the KamLAND experiment [14] as an example. The figure compares actual quantum 
(a)

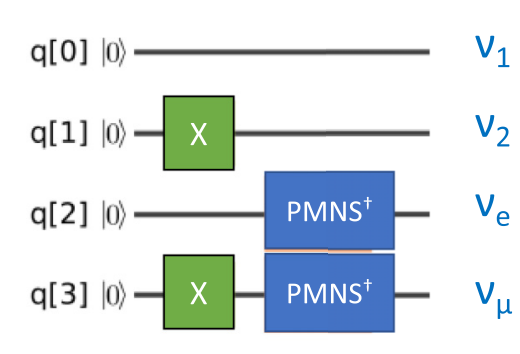

(d) (c) (b)

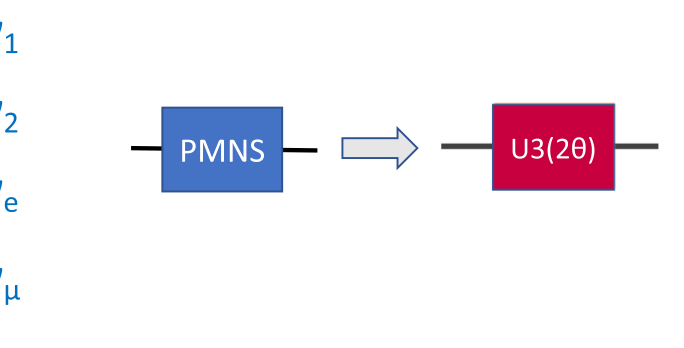

d)
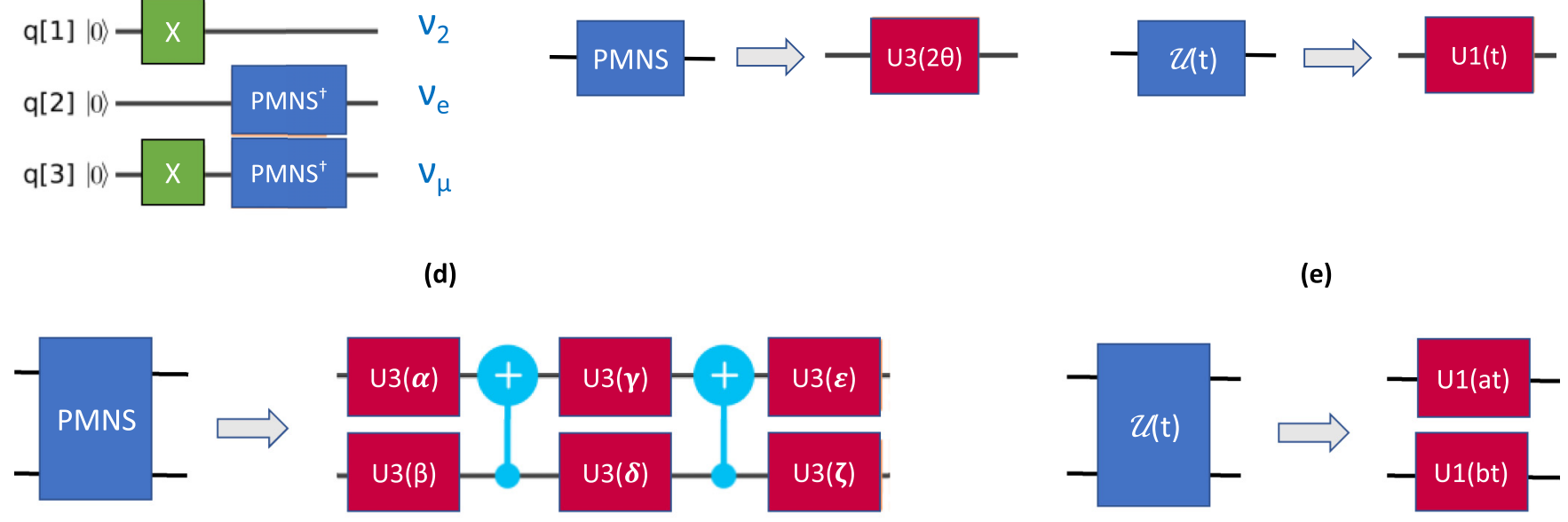

(e)

FIG. 1. (a) Preparation of neutrino flavor and mass basis states in the $2 \times 2$ quantum computation, (b) PMNS gate, and (c) time-evolution gates. (d) PMNS gate and (c) time-evolution gate in the $3 \times 3$ quantum computation.

computations, calculated on the IBM quantum processor (squares); simulated runs of the quantum computer, which represent the same operations performed without decoherence or errors (circles); and the standard two-flavor oscillation formula (lines). The quantum evolution matches very well with expectations from both theory and quantum simulation.

For this circuit and others described in this paper we have chosen gate arrangements that are simple and intuitive, mirroring the quantum operations involved in physical neutrino oscillations. It is however clear that this physically motivated sequence is not necessarily the most efficient way

(a)

(b)

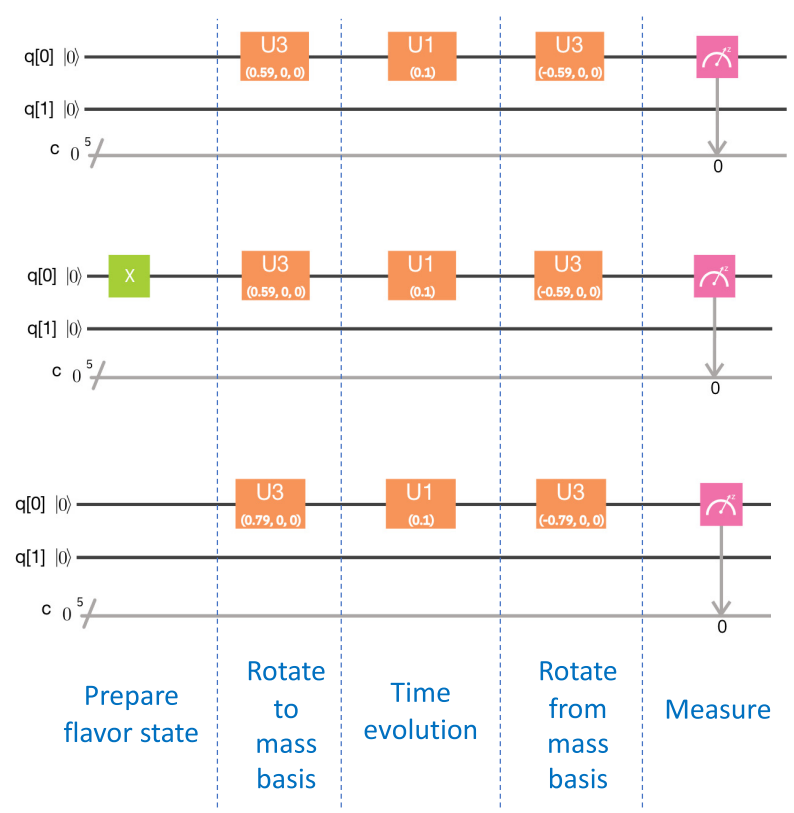

FIG. 2. Quantum circuits embodying two-flavor neutrino oscillation for (a) $v_{e}$ disappearance, (b) $v_{\mu} \rightarrow v_{e}$, and (c) $v_{\mu} \rightarrow v_{\tau}$. of performing the relevant unitary operation on a quantum computer. The three gates of Fig. 1 that represent flavor rotation, time evolution, and inverse flavor rotation could, for example, be combined into a single $U(3)$ gate. Similar simplifications are possible for the other circuits presented in this paper. Since our goal in this work is to illustrate the physical encoding of the neutrino oscillations system into a quantum computer, we have opted for the more intuitive, physically motivated circuit layouts throughout.

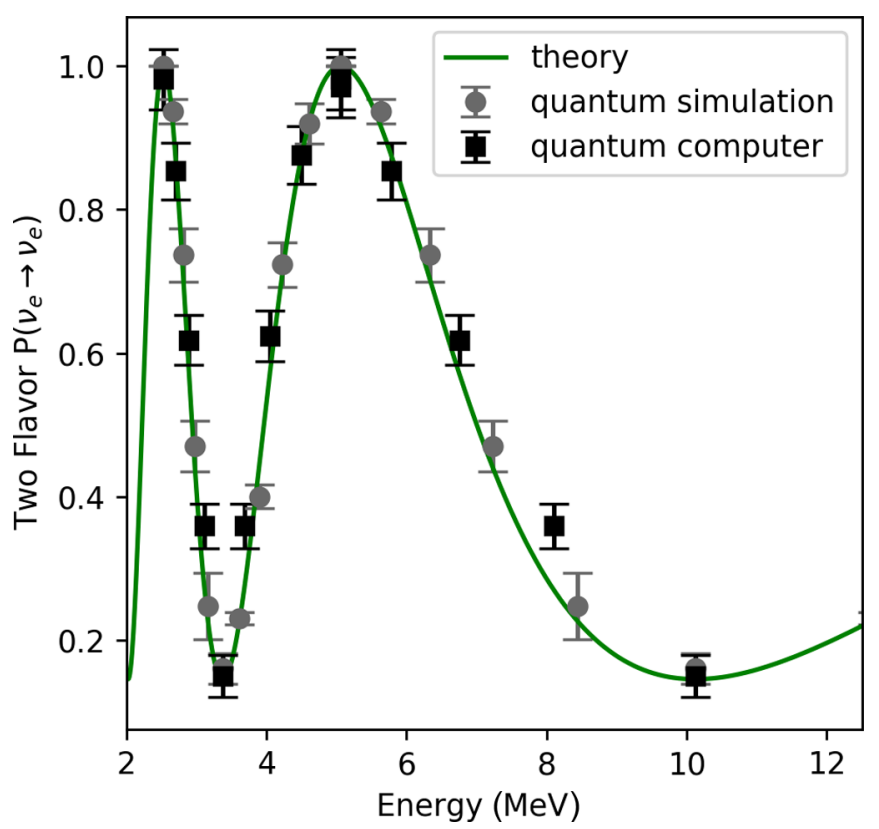

FIG. 3. Two-flavor electron-neutrino survival probability as a function of the neutrino energy. The green line shows the theoretical calculation using a classical computer. The black circle markers indicate a quantum computer simulation and the black square markers are the output of the IBM Q quantum computer. 


\section{THREE-FLAVOR NEUTRINO OSCILLATION}

A three-flavor neutrino oscillation involves a Hilbert space of dimension 3 , requiring more than one qubit. The minimal representation can be encoded on two qubits, via a basis definition such as

$$
\begin{array}{ll}
|00\rangle \rightarrow\left|v_{1}\right\rangle=(1,0,0,0), & |01\rangle \rightarrow\left|v_{2}\right\rangle=(0,1,0,0), \\
|10\rangle \rightarrow\left|v_{3}\right\rangle=(0,0,1,0), & |11\rangle \rightarrow\left|v_{X}\right\rangle=(0,0,0,1) .
\end{array}
$$

There is one redundant basis state $\left|v_{X}\right\rangle$ in this representation. This could represent a fourth neutrino flavor in models with sterile neutrinos, but for the present example we will consider it as physically decoupled, and thus unphysical. As in the two-flavor case, to prepare a flavor state, we must apply the PMNS operation to an initial state in the computational basis. Unlike in the two-neutrino example, however, creating a set of quantum gates to implement the PMNS operation on two entangled qubits is nontrivial. A real unitary two-qubit gate requires at least two CNOT and 12 elementary gates [46] for an entirely general representation. Constraints on the PMNS matrix due to rephasing invariance may be expected to allow for a more compact representation. Following exploration of several possibilities, we constructed a parametrizable set of six real $U 3$ gates acting on two qubits $A$ and $B$, with two interspersed CNOT gates to reproduce the PMNS operation. To fix the free parameters of this arrangement, we map the circuit onto matrix multiplication in the computational basis and perform a numerical fit to match its entries to the experimentally determined PMNS matrix elements [47]. We fit for the PMNS matrix and invert the gate arrangement for $\mathrm{PMNS}^{\dagger}$. The PMNS and PMNS ${ }^{\dagger}$ operations are thus constructed as

$$
\begin{aligned}
\mathrm{PMNS}= & U 3_{A}(\epsilon) U 3_{B}(\zeta) \mathrm{CNOT}_{A B} \\
& \times U 3_{A}(\gamma) U 3(\delta)_{B} \mathrm{CNOT}_{A B} U 3_{A}(\alpha) U 3(\beta)_{B}, \\
\mathrm{PMNS}^{\dagger}= & U 3_{A}(-\alpha) U 3_{B}(-\beta) \mathrm{CNOT}_{A B} \\
& \times U 3_{A}(-\gamma) U 3_{B}(-\delta) \mathrm{CNOT}_{A B} U 3_{A}(-\epsilon) U 3(-\zeta)_{B} .
\end{aligned}
$$

The best-fit parameters $\alpha, \beta, \gamma, \delta, \epsilon$, and $\zeta$ (primed and unprimed) reproduce the measured PMNS and $\mathrm{PMNS}^{\dagger}$ elements within one part in $10^{6}$ when no $C P$ violating phase is present, comfortably within experimental uncertainty. This parametrization can be extended with two additional $U 3$ gates and a CNOT in order to incorporate a Dirac $C P$ phase with similar accuracy. These parameters are tabulated in Appendix B. The PMNS gate decomposition in terms of component gates is shown diagrammatically in Fig. 1(d).

Once the initial flavor state is prepared, the time-evolution operation $\mathcal{U}$ must be implemented. This is represented in the computational basis by

$$
\mathcal{U}(t)=\exp \left[i \operatorname{diag}\left(0, \Delta m_{12}^{2} \frac{t}{2 E \hbar}, \Delta m_{13}^{2} \frac{t}{2 E \hbar}, \Phi\right)\right],
$$

where $\Phi$ is an arbitrary phase that can be picked for convenience, since the fourth basis state is unobservable. A straightforward choice that can be implemented as one-qubit gates acting on $A$ and $B$ is

$$
\mathcal{U}(t)=S_{A}\left(i \Delta m_{13}^{2} \frac{t}{2 E \hbar}\right) S_{B}\left(i \Delta m_{12}^{2} \frac{t}{2 E \hbar}\right),
$$

where $S_{A}$ and $S_{B}$ are the $S$ gates for qubits $A$ and $B$, respectively.

The complete quantum circuit for the oscillation calculation in the three-neutrino space comprises state preparation, time evolution, and flavor measurement and is shown in Appendix A, Fig. 6. This circuit can be run repeatedly to prepare qubits in flavor eigenstates, time evolve them, and measure their flavor after propagation in order to establish oscillation probabilities. Since this is a substantially more complex circuit than the two-flavor case, gate errors and read errors are expected to be more prevalent. We correct for the effects of read errors in the final oscillation probability by applying an inverted error matrix in the computational basis, which accounts for decoherence and read errors in a statistical manner, based on qubit readout accuracy measured using runs with $L / E=0$. More details on this procedure can be found in Appendix C.

Figure 4 shows two example calculations of three-flavor oscillation probabilities given an initial muon neutrino beam. Figure 4(a) shows calculations at smaller $L / E$ where the oscillation is effectively a two-flavor system. Figure 4(b) shows the behavior near the first oscillation maximum where three flavors $v_{e}, v_{\mu}$, and $v_{\tau}$ are participating strongly. Good agreement with theory is observed in both regimes. In both cases, the electron flavor is slightly overrepresented, potentially due to read and gate errors that are not entirely symmetrically distributed between flavors. The size of the effect is comparable to the statistical and systematic uncertainty, which receives contributions from (i) accumulated gate errors, based on the ibmqx 2 spec of $\sim 10^{-3}$ per gate added in quadrature over 50 gates, and (ii) statistical uncertainty, from the finite number of evolutions (1024) used to establish the oscillation probability. After running the simulation and applying a statistical readout error mitigation tuned on zero-time simulations (explained in Appendix C), strong agreement between the quantum and classical computations are obtained.

\section{NEUTRINO OSCILLATIONS WITH NEW PHYSICS}

In addition to standard neutrino oscillations, beyondstandard-model (BSM) effects that have been sought in neutrino oscillation experiments can be incorporated into the quantum circuit straightforwardly either by (a) extending the PMNS matrix and time-evolution operator to higher dimensionality or (b) introducing new effects in the time-evolution term. Here we briefly review a few of these scenarios.

\section{A. Sterile neutrinos}

As we have seen, the incorporation of at least one additional basis state within the Hilbert space is mandatory, given a two-qubit realization. To use this state to represent an oscillating forth neutrino, as suggested by short baseline neutrino anomalies [48-51], two adjustments are required: (i) extension of the PMNS matrix to mixing in four dimensions, which is already achievable in our present parametrization, given 


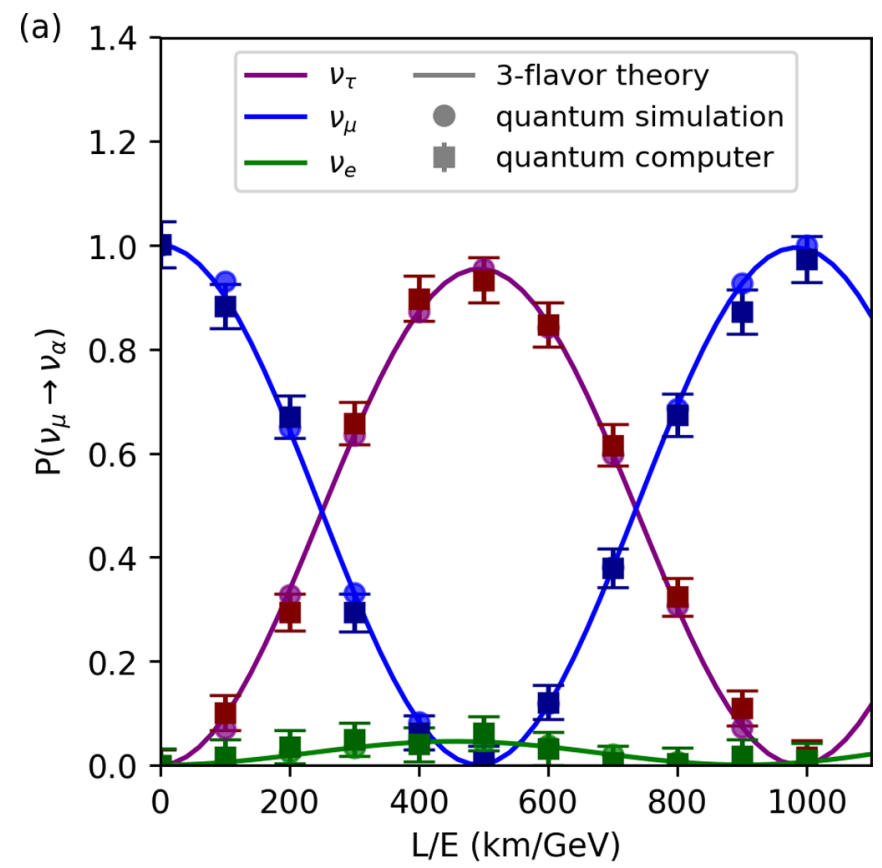

(b)

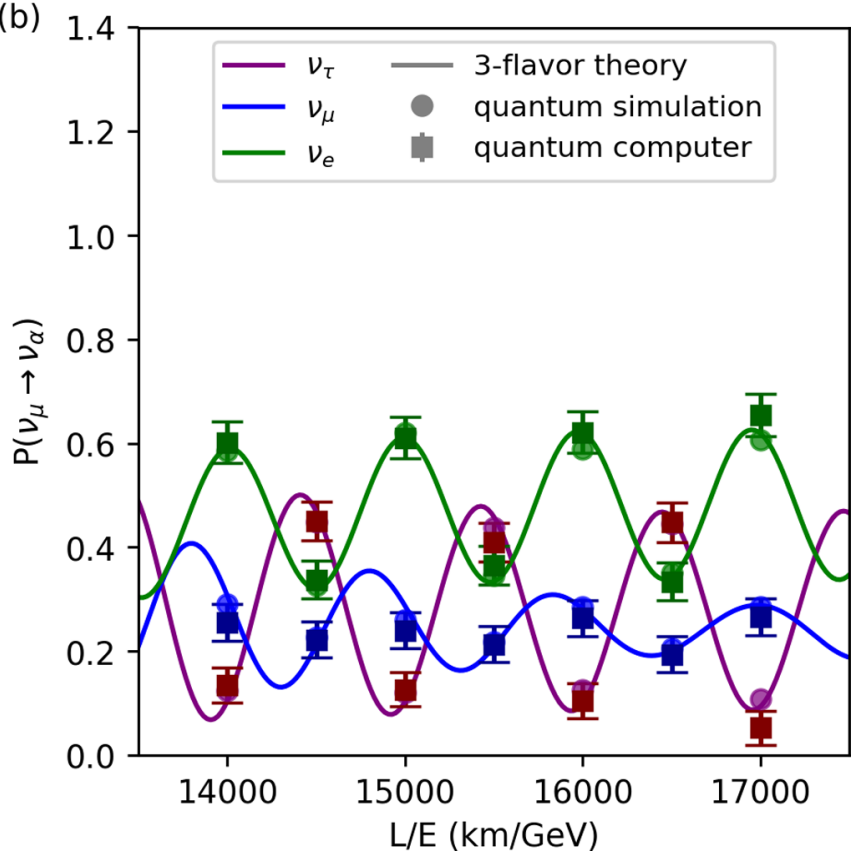

FIG. 4. Calculations of three-flavor neutrino oscillations evaluated using a quantum computer (squares), quantum computer simulator (circles), and theory (lines). The quantum computer results have been corrected for gate read errors based on the matrix $M$ described in the text. The two plots (a) and (b) show three-flavor oscillation probabilities calculated in two characteristic $L / E$ ranges.

appropriate gate coefficients, and (ii) independent control of the phase of the $\left|v_{4}\right\rangle=|11\rangle$ mass state in the time-evolution operator. This is necessarily an operation that involves entangling the two qubits and so cannot be implemented on singlequbit gates only. A circuit that produces the required time evolution (an independently specified phase on each of $|01\rangle$, (a)

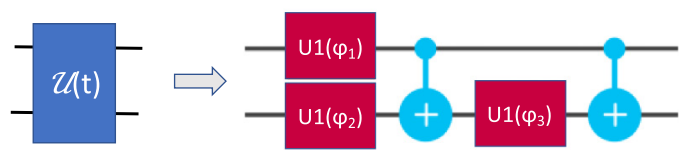

(b)

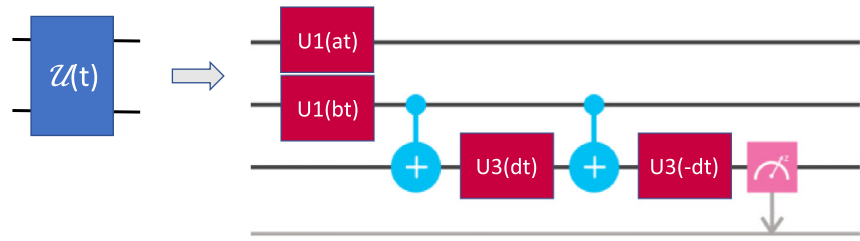

FIG. 5. Quantum circuits for two BSM oscillation scenarios: (a) sterile neutrino oscillations and (b) anomalous decoherence in the mass basis.

$|10\rangle$, and $|11\rangle$ ) is shown in Fig. 5(a). This circuit, configured with the parameters

$$
\begin{aligned}
\phi_{1} & =\frac{1}{4 E \hbar}\left(\Delta m_{12}^{2}-\Delta m_{13}^{2}+\Delta m_{14}^{2}\right), \\
\phi_{2} & =\frac{1}{4 E \hbar}\left(\Delta m_{12}^{2}+\Delta m_{13}^{2}-\Delta m_{14}^{2}\right), \\
\phi_{3} & =\frac{1}{4 E \hbar}\left(-\Delta m_{12}^{2}+\Delta m_{13}^{2}+\Delta m_{14}^{2}\right),
\end{aligned}
$$

will achieve the necessary four-state time evolution needed to implement quantum simulations of the four-flavor neutrino system extended for a single sterile neutrino.

\section{B. Nonstandard interactions and matter effects}

The modeling of either standard [7,8] or nonstandard $[52,53]$ matter effects, with or without violations of Lorentz symmetry, can be incorporated without changing the threeflavor oscillation quantum circuit by adjustment of the input parameters that describe the PMNS and time-evolution operations in the modified matter basis. A discussion of the parametrizations that incorporate these effects is given in Appendixes D and E.

\section{Decoherence}

Decoherence is a nonstandard neutrino oscillation effect $[20,54,55]$ that is often considered in connection with quantum gravity or space-time foam models [56]. In decoherence scenarios, development of entanglements between parts of the neutrino wave function and an external environment lead to partial collapse of the wave function and suppression of oscillations. Decoherence can be manifest in various bases, depending on the degrees of freedom within the neutrino subsystem that the environment entangles. Figure 5(b) illustrates a quantum circuit that implements decoherence in neutrino oscillations via generation of entanglements in the mass basis for small $d t$. In this circuit, an auxiliary qubit representing the environment is initialized to zero and acquires a small and time-dependent admixture of the $|1\rangle$ basis state, if and only if the second neutrino qubit is in a $|1\rangle$ state. This entanglement between the system and the ancilla qubit 
acts to suppress coherence in the off-diagonal elements of the neutrino system density matrix. The process of continuous measurement during evolution for large $d t$ is approximated by repeated units of time evolution, entanglement, and measurement, resetting the ancilla to zero after each time step. In such a scheme, entanglement is developed between the system and ancilla, suppressing the off-diagonal system density matrix elements. This entanglement is then conveyed to the outside world via measurement. The process of measurement does not itself generate decoherence, but allows the ancilla to be disentangled and reset to zero for the next block of time evolution and further suppression of the off-diagonal system density matrix elements. Such a scheme could model gravitational decoherence, or flavor change through wave-packet separation given a normal ordering of neutrino masses (with one mass state much heavier than the others).

\section{CONCLUSION}

We have demonstrated a quantum mechanical simulation of neutrino oscillations on a quantum computer, using both two-flavor and three-flavor systems. The two-flavor system has an almost trivial realization in the two-dimensional Hilbert space of a single qubit, with implementation of PMNS and time-evolution gates using individual single-qubit gates. The three-neutrino system, on the other hand, requires a more complicated quantum circuit, involving the entanglement of two qubits to produce a Hilbert space of four dimensions. A subspace of three of these states is used for the calculation. Our implementation of the $3 \times 3$ PMNS matrix on the twoqubit space in terms of six general rotation gates and two CNOT gates provides an accurate and economical realization of the PMNS operation within a quantum circuit. By choosing a phase convention such that phases are measured relative to $v_{1}$ and allowing for phase freedom in the unphysical fourth basis state, the time-evolution operator can be implemented using two single-qubit gates.

Quantum calculations using both the two- and three-flavor systems agree with theoretical expectations for the neutrino oscillation probability within systematic and statistical uncertainty. Although in agreement, the calculation presented here is characteristically different from the classical computation of oscillation probabilities, since the qubits act as direct quantum analogs to the evolving neutrino flavor wave function. The system is prepared coherently, evolves forward in time unitarily, and has its wave function collapsed to measure the final flavor oscillations, just as in a neutrino oscillation experiment.

Analogs of real quantum systems inside quantum processors such as the one presented in this paper may eventually enable computations that surpass the capabilities of their classical counterparts. This is especially likely for strongly coupled or highly entangled systems, such as collectively oscillating neutrinos in supernovae. Understanding how to translate simple and well-understood calculations into quantum circuits is a necessary step toward realizing this goal. In this work, we have presented one such example, creating an analog to two- and three-flavor neutrino oscillations inside a publicly accessible quantum processor.

\section{ACKNOWLEDGMENTS}

We thank Jean DeMerit, Kareem R. H. A. M. Farrag, Roxanne Guenette, Jonathan Asaadi, and Peter Denton for providing insightful comments. B.J.P.J. was supported by the Department of Energy under Awards No. DE-SC0019054 and No. DE-SC0019223. C.A.A. was supported by U.S. National Science Foundation Grant No. PHY-1801996. B.J.P.J. and C.A.A. thank the organizers of the workshop "New Opportunities at the Next Generation Neutrino Experiments" at the University of Texas at Arlington, where this work was completed. We thank IBM for making the public quantum processor available to the world, an invaluable resource as we prepare to move towards the quantum revolution. We acknowledge use of the IBM Q for this work. The views expressed are those of the authors and do not reflect the official policy or position of IBM or the IBM Q team.

\section{APPENDIX A: RUNNING QUANTUM COMPUTATION ON IBM Q}

Computations were run on two IBM publicly accessible quantum computers ibmqx2 (Yorktown). The two least-readerror-prone qubits on this five-qubit machine that could be connected by the appropriate logic gates within the allowable topology were chosen to support the computational basis. At the time of writing these were qubits 0 and 2 , with reported gate errors of $0.77 \times 10^{-3}$ and $1.03 \times 10^{-3}$ and read errors of $7.6 \%$ and $2.9 \%$, respectively, and a multiqubit read error of $2.21 \%$. Some supplementary calculations were also run on IBM Q ourense.

It is difficult to convert such gatewise error specifications into an expected calculational accuracy, so we instead opted to measure the accuracy directly by running $L / E=0$ simulations. Some contribution to the error budget is expected from errors that accumulate only when the time evolves a finite amount, so we conservatively associate an additional contribution for gate error on top of that measured from unoscillated points. The complete quantum circuit as implemented on ibmqx 2 is shown in Fig. 6. This was run with 1024 shots to establish the survival probability, and the statistical error associated with this count is included in our uncertainty budget.

As well as access to the quantum processor, the IBM website offers a quantum simulator tool to simulate the circuit before running it. In all cases, simulations agreed near perfectly with theoretical expectations. This suggests that wherever the data show small differences from theoretical expectations, these are to be attributed to the imperfections of the quantum processor.

\section{APPENDIX B: FITTING THE PMNS MATRIX GATE PARAMETERS}

The fit to the PMNS matrix is made by gradient minimization over six parameters. We minimize the sum of squared residuals of each of the elements of the matrix. The final fit converges to the true PMNS matrix within one part in $10^{6}$ for every element, comfortably within experimental uncertainty. The input values of the PMNS matrix used in the fit are from [47] and are 


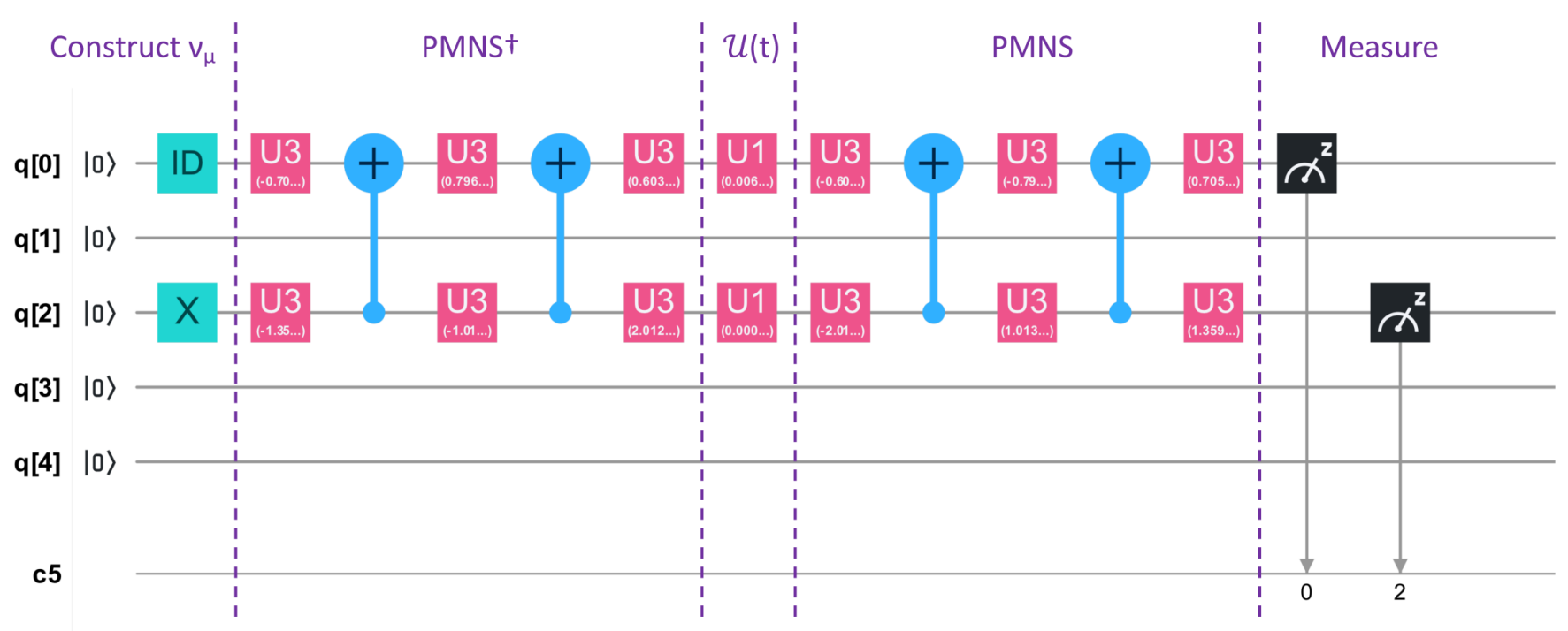

FIG. 6. Three-flavor neutrino oscillation experiment as run on the IBM quantum computer.

$$
U_{\text {PMNS }}=\left(\begin{array}{cccc}
0.821427 & 0.550313 & 0.149708 & 0 \\
-0.481513 & 0.528538 & 0.699138 & 0 \\
0.305618 & -0.646377 & 0.699138 & 0 \\
0 & 0 & 0 & 1
\end{array}\right)
$$

The bottom row represents a decoupled unphysical state, but is necessary to span the four-dimensional Hilbert space represented by two qubits. The best-fit parameters from fitting the PMNS and PMNS ${ }^{\dagger}$ matrices in the computational basis are

$$
\begin{aligned}
& \alpha=-0.6031, \quad \beta=-2.0125, \quad \gamma=0.7966, \\
& \delta=1.0139, \quad \epsilon=0.7053, \quad \zeta=1.3599 .
\end{aligned}
$$

This gate configuration will apply the PMNS rotation to any state prepared in the mass basis, as defined in this work:

$$
M=\left(\begin{array}{cccc}
\left(1-f_{1}\right)\left(1-f_{2}\right)+f_{1}^{2}+f_{2}^{2} & \left(1-f_{1}\right) f_{2}-f_{2}^{2} & \left(1-f_{2}\right) f_{1}-f_{1}^{2} & f_{1} f_{2} \\
\left(1-f_{1}\right) f_{2}-f_{2}^{2} & \left(1-f_{1}\right)\left(1-f_{2}\right)+f_{1}^{2}+f_{2}^{2} & f_{1} f_{2} & \left(1-f_{2}\right) f_{1}-f_{1}^{2} \\
\left(1-f_{2}\right) f_{1}-f_{1}^{2} & f_{1} f_{2} & \left(1-f_{1}\right)\left(1-f_{2}\right)+f_{1}^{2}+f_{2}^{2} & \left(1-f_{1}\right) f_{2}-f_{2}^{2} \\
f_{1} f_{2} & \left(1-f_{2}\right) f_{1}-f_{1}^{2} & \left(1-f_{1}\right) f_{2}-f_{2}^{2} & \left(1-f_{1}\right)\left(1-f_{2}\right)+f_{1}^{2}+f_{2}^{2}
\end{array}\right) .
$$

\section{APPENDIX C: CORRECTING FOR BIT FLIPS AND DECOHERENCE}

Some random qubit readout errors are naturally expected for any quantum computation. However, we can correct our final distributions against some of these information losses statistically. We consider that there is some average probability $f_{1}$ or $f_{2}$ for either of the qubits to be flipped leading to an incorrect flavor measurement, which is approximately uniform across circuits. Then the effect on the final distribution of events in $\left(v_{e}, v_{\mu}, v_{\tau}, v_{X}\right)$ space is to multiply final-state distributions by a matrix $M$, given in Appendix B.

The form of $M$ can be understood by considering that spurious transitions $v_{\mu} \leftrightarrow v_{e}, v_{\tau} \leftrightarrow v_{e}, v_{\tau} \leftrightarrow v_{s}$, and $v_{\mu} \leftrightarrow v_{s}$ require only one qubit flip in the final read, whereas spurious transitions $v_{e} \leftrightarrow v_{s}$ and $v_{\mu} \leftrightarrow v_{\tau}$ require two. Here $f_{1}$ and $f_{2}$, the rates of bit flips in qubits 1 and 2, can be measured by examining the rate of spurious transitions at $L / E=0$, where no physical oscillation effects are expected, and any transformation must be associated with spurious bit flips. The quantum simulator predicts zero transformation at $L / E=0$, whereas we find that the quantum computer gives some random transformations, consistent with $f_{1} \sim 13 \%$ and $f_{2} \sim 3 \%$. With $f$ measured, we can invert $M$ and apply this inverted matrix to correct the final probability distributions. This correction is applied in order to obtain our final comparison of the data and theory.

\section{APPENDIX D: INCORPORATION OF MATTER POTENTIALS AND NONSTANDARD INTERACTIONS}

When neutrinos travel through a medium they experience a potential produced by coherent forward scattering with electrons, protons, and neutrons. The potential sourced by protons and neutrons is the same for all neutrino flavors and thus induces an overall phase in the neutrino system evolution. The electron charged-current potential is flavor asymmetric, producing an observable modification in the neutrino 
oscillation probability. The matter potential can be written in the flavor basis as

$$
V_{m}=\sqrt{2} G_{F}\left(\begin{array}{ccc}
N_{e} & 0 & 0 \\
0 & 0 & 0 \\
0 & 0 & 0
\end{array}\right),
$$

where $G_{F}$ is the Fermi constant and $N_{e}$ is the electron number density. Then the total neutrino Hamiltonian can be written as

$$
H=H_{\mathrm{vac}}+V_{m}=U_{m}^{\dagger} \Delta U_{m} .
$$

The Hamiltonian can be diagonalized by a unitary transformation $U_{m}$ that relates the flavor basis to the Hamiltonian eigenstates and a diagonal matrix $\Delta$, which contains the energy eigenvalues. In a two-flavor system, $U_{m}$ can be written as a $2 \times 2$ rotation matrix analogous to vacuum case 3 , where the rotation angle is given by

$$
\theta_{m}=\arctan \frac{\Delta \sin ^{2} 2 \theta}{\Delta \cos 2 \theta_{0}-\sqrt{2} G_{F} N_{e}},
$$

where $\Delta=\Delta m^{2} / 2 E$ and $\theta_{0}$ is the vacuum mixing angle. The relevant Hamiltonian eigenvalue difference is given by

$$
\lambda=\sqrt{\left(\Delta \cos 2 \theta_{0}-\sqrt{2} G_{F} N_{e}\right)^{2}+\Delta^{2} \sin ^{2} 2 \theta_{0}} .
$$

Thus the matter modification does not require a new quantum circuit; we can simply replace the vacuum mixing angle and eigenvalue for the expressions given above. For the case of the three-neutrino scenario, exact expressions for the effective mixing angles and eigenvalues are lengthy, but can be readily found by numerical diagonalization.

Effects of the standard neutrino matter potential have been observed with natural sources, e.g., in solar neutrino experiments, and with human-made sources, e.g., in accelerator neutrinos experiments. Deviations from the standard potential can be due to new forces that manifest themselves as vector or scalar interactions. These have been constrained by searches of anomalous neutrino flavor changing and more recently in coherent-scattering neutrino experiments. The status of recent constraint on nonstandard interactions can be found in Ref. [57], where the constraints are given relative to the weak-force strength $G_{F}$ and flavor-dependent coefficients $\epsilon_{\alpha \beta}$. Depending on the target and the flavor structure these are constrained from $O(1 \%)$ to $O(10 \%)$. The effects of nonstandard vector interactions can also be calculated using a quantum processor by using the PMNS gate with effective matter potential mixing angles. The appropriate angles and eigenvalues can be determined by diagonalizing the Hamiltonian in Eq. (D2) where one ought to replace $V_{m}$ by

$$
V_{m}^{n s i}=\sqrt{2} G_{F} N_{e}\left(\begin{array}{ccc}
1+\epsilon_{e e} & 0 & 0 \\
0 & 0 & 0 \\
0 & 0 & 0
\end{array}\right) .
$$

\section{APPENDIX E: INCORPORATION OF LORENTZ VIOLATION}

The standard model (SM) of particle physics [58] can be thought as an effective field theory towards a grand unified theory of nature [59]. In many extensions of the SM, Lorentz symmetry is broken. Neutrinos, as natural interferometers, are extremely sensitive to high-scales where Lorentz violation (LV) may be manifest. In fact, neutrinos have some of the strongest constraints on LV nonrenormalizable operators [24]. Calculations of oscillation probabilities in the presence of LV can be performed using the quantum circuits presented in this paper. Lorentz violation can be incorporated in the neutrino Hamiltonian as

$$
H=H_{\mathrm{vac}}+\tilde{a}^{3}+\tilde{c}^{4} E+\tilde{a}^{5} E^{2}+\tilde{c}^{6} E^{3}+\cdots,
$$

where $\tilde{a}^{d}\left(\tilde{c}^{d}\right)$ is a matrix that contains the strength of interaction between the neutrino and a Lorentz violating field produced by a CPT even (odd) effective operator of dimension $d$. Similarly to the case of matter interactions, this Hamiltonian can be diagonalized numerically in order to obtain appropriate effective mixing angles and frequencies. One can then use the PMNS quantum gate and evolution operators discussed in the main text.
[1] T. Kajita, Nobel Lecture: Discovery of atmospheric neutrino oscillations, Rev. Mod. Phys. 88, 030501 (2016).

[2] M. Gell-Mann, P. Ramond, and R. Slansky, in Proceedings of the Stony Brook Supergravity Workshop, edited by P. van Nieuwenhuizen and D. Z. Freedman (North-Holland, Amsterdam, 1979), pp. 315-321.

[3] T. Yanagida, in Proceedings of the Workshop on the Unified Theory and the Baryon Number in the Universe, Tsukuba, 1979, edited by O. Sawada and A. Sugamoto (KEK, Tsukuba, 1979).

[4] R. N. Mohapatra and G. Senjanović, Neutrino masses and mixings in gauge models with spontaneous parity violation, Phys. Rev. D 23, 165 (1981).

[5] M. Fukugita and T. Yanagida, Baryogenesis without grand unification, Phys. Lett. B. 174, 45 (1986).

[6] K. Nakamura et al., Review of particle physics, J. Phys. G 37, 075021 (2010).

[7] L. Wolfenstein, Neutrino oscillations in matter, Phys. Rev. D 17, 2369 (1978); R. A. Eramzhyan, M. Gmitro, R. A. Sakaev, and L. A. Tosunjan, Towards a better understanding of ${ }^{16} \mathrm{O}$ nuclear structure: Muon capture and radiative pion capture reactions, Nucl. Phys. A 290, 294 (1977).

[8] S. P. Mikheyev and A. Yu. Smirnov, Resonant amplification of $v$ oscillations in matter and solar-neutrino spectroscopy, Il Nuovo Cimento C 9, 17 (1986).

[9] C. Giunti and C. W. Kim, Fundamentals of Neutrino Physics and Astrophysics (Oxford University Press, Oxford, 2007).

[10] P. Adamson et al. (MINOS Collaboration), Improved Search for Muon-Neutrino to Electron-Neutrino Oscillations in MINOS, Phys. Rev. Lett. 107, 181802 (2011).

[11] P. Adamson et al. (NOvA Collaboration), First Measurement of Electron Neutrino Appearance in NOvA, Phys. Rev. Lett. 116, 151806 (2016).

[12] Y. Fukuda et al. (Super-Kamiokande Collaboration), Evidence for Oscillation of Atmospheric Neutrinos, Phys. Rev. Lett. 81, 1562 (1998).

[13] M. G. Aartsen et al. (IceCube Collaboration), Searches for Sterile Neutrinos with the IceCube Detector, Phys. Rev. Lett. 117, 071801 (2016). 
[14] K. Eguchi et al. (KamLAND Collaboration), First Results from KamLAND: Evidence for Reactor Antineutrino Disappearance, Phys. Rev. Lett. 90, 021802 (2003).

[15] F. P. An et al., Observation of Electron-Antineutrino Disappearance at Daya Bay, Phys. Rev. Lett. 108, 171803 (2012).

[16] R. Davis, Jr., D. S. Harmer, and K. C. Hoffman, Search for Neutrinos from the Sun, Phys. Rev. Lett. 20, 1205 (1968).

[17] J. A. Formaggio, D. I. Kaiser, M. M. Murskyj, and T. E. Weiss, Violation of the Leggett-Garg Inequality in Neutrino Oscillations, Phys. Rev. Lett. 117, 050402 (2016).

[18] J. S. Bell, in On the Foundations of Quantum Mechanics, edited by M. Bell and M. Veltman (World Scientific, Singapore, 2001), pp. 1-6.

[19] E. K. Akhmedov and A. Y. Smirnov, Paradoxes of neutrino oscillations, Phys. At. Nucl. 72, 1363 (2009).

[20] E. Lisi, A. Marrone, and D. Montanino, Probing Possible Decoherence Effects in Atmospheric Neutrino Oscillations, Phys. Rev. Lett. 85, 1166 (2000).

[21] G. L. Fogli, E. Lisi, A. Marrone, and D. Montanino, Status of atmospheric neutrino $v_{\mu} \rightarrow v_{\tau}$ oscillations and decoherence after the first K2K spectral data, Phys. Rev. D 67, 093006 (2003).

[22] V. A. Kostelecky and M. Mewes, Lorentz and $C P T$ violation in neutrinos, Phys. Rev. D 69, 016005 (2004).

[23] J. R. Ellis, N. Harries, A. Meregaglia, A. Rubbia, and A. Sakharov, Probes of Lorentz violation in neutrino propagation, Phys. Rev. D 78, 033013 (2008).

[24] M. G. Aartsen et al. (IceCube Collaboration), Neutrino interferometry for high-precision tests of Lorentz symmetry with IceCube, Nat. Phys. 14, 961 (2018).

[25] N. E. Mavromatos, $C P T$ violation and decoherence in quantum gravity, Lect. Notes Phys. 669, 245 (2005).

[26] J. Alfaro, H. A. Morales-Tecotl, and L. F. Urrutia, Quantum Gravity Corrections to Neutrino Propagation, Phys. Rev. Lett. 84, 2318 (2000).

[27] M. Gasperini, Testing the principle of equivalence with neutrino oscillations, Phys. Rev. D 38, 2635 (1988).

[28] B. J. P. Jones, Dynamical pion collapse and the coherence of conventional neutrino beams, Phys. Rev. D 91, 053002 (2015).

[29] L. K. Grover, A fast quantum mechanical algorithm for database search, in Proceedings of the 28th Annual ACM Symposium on Theory of Computing, STOC '96, Philadelphia, Pennsylvania, 1996 (ACM, New York, 1996), pp. 212-219.

[30] P. W. Shor, Polynomial time algorithms for prime factorization and discrete logarithms on a quantum computer, SIAM J. Sci. Stat. Comput. 26, 1484 (1997).

[31] R. P. Feynman, Simulating physics with computers, Int. J. Theor. Phys. 21, 467 (1982).

[32] I. M. Georgescu, S. Ashhab, and F. Nori, Quantum simulation, Rev. Mod. Phys. 86, 153 (2014).

[33] C. W. Bauer, W. A. de Jong, B. Nachman, and D. Provasoli, A quantum algorithm for high energy physics simulations, arXiv:1904.03196.

[34] A. Y. Wei, P. Naik, A. W. Harrow, and J. Thaler, Quantum algorithms for jet clustering, arXiv:1908.08949.

[35] I. C. Cloët, M. R. Dietrich, J. Arrington et al., in Opportunities for Nuclear Physics \& Quantum Information Science, Proceed- ings of the Workshop on Intersections between Nuclear Physics and Quantum Information, Lemont, 2018, edited by I. C. Cloët and M. R. Dietrich, U.S. DOE report (unpublished), available at https://arxiv.org/pdf/1903.05453.pdf.

[36] I. Buluta and F. Nori, Quantum simulators, Science 326, 108 (2009).

[37] U.-J. Wiese, Towards quantum simulating QCD, Nucl. Phys. A 931, 246 (2014).

[38] G. Sigl and G. Raffelt, General kinetic description of relativistic mixed neutrinos, Nucl. Phys. B 406, 423 (1993).

[39] H. Duan, G. M. Fuller, and Y.-Z. Qian, Collective neutrino oscillations, Annu. Rev. Nucl. Part. Sci. 60, 569 (2010).

[40] S. Biondini et al., Status of rates and rate equations for thermal leptogenesis, Int. J. Mod. Phys. A 33, 1842004 (2018).

[41] S. Blanchet, P. Di Bari, D. A. Jones, and L. Marzola, Leptogenesis with heavy neutrino flavours: From density matrix to Boltzmann equations, J. Cosmol. Astropart. Phys. 1301, 041 (2013).

[42] P. Hernández, M. Kekic, J. López-Pavón, J. Racker, and J. Salvado, Testable baryogenesis in seesaw models, J. High Energy Phys. 08 (2016) 157.

[43] C. A. Argüelles Delgado, J. Salvado, and C. N. Weaver, A simple quantum integro-differential solver (SQuIDS), Comput. Phys. Commun. 196, 569 (2015).

[44] G. B. Lesovik, I. A. Sadovskyy, M. V. Suslov, A. V. Lebedev, and V. M. Vinokur, Arrow of time and its reversal on the IBM quantum computer, Sci. Rep. 9, 4396 (2019).

[45] C. Giunti, No effect of Majorana phases in neutrino oscillations, Phys. Lett. B 686, 41 (2010).

[46] F. Vatan and C. Williams, Optimal quantum circuits for general two-qubit gates, Phys. Rev. A 69, 032315 (2004).

[47] I. Esteban, M. C. Gonzalez-Garcia, A. Hernandez-Cabezudo, M. Maltoni, and T. Schwetz, Global analysis of three-flavour neutrino oscillations: Synergies and tensions in the determination of $\theta_{2} 3, \delta_{C} P$, and the mass ordering, J. High Energy Phys. 01 (2019) 106.

[48] A. A. Aguilar-Arevalo et al. (MiniBooNE Collaboration), Significant Excess of Electronlike Events in the MiniBooNE ShortBaseline Neutrino Experiment, Phys. Rev. Lett. 121, 221801 (2018).

[49] J. Kopp, P. A. N. Machado, M. Maltoni, and T. Schwetz, Sterile neutrino oscillations: The global picture, J. High Energy Phys. 05 (2013) 050.

[50] G. Mention, M. Fechner, T. Lasserre, T. A. Mueller, D. Lhuillier, M. Cribier, and A. Letourneau, Reactor antineutrino anomaly, Phys. Rev. D 83, 073006 (2011).

[51] A. Aguilar et al. (LSND Collaboration), Evidence for neutrino oscillations from the observation of $\bar{v}_{e}$ appearance in a $\bar{v}_{\mu}$ beam, Phys. Rev. D 64, 112007 (2001).

[52] S. Antusch, J. P. Baumann, and E. Fernandez-Martinez, Nonstandard neutrino interactions with matter from physics beyond the standard model, Nucl. Phys. B 810, 369 (2009).

[53] S. Davidson, C. Pena-Garay, N. Rius, and A. Santamaria, Present and future bounds on nonstandard neutrino interactions, J. High Energy Phys. 03 (2003) 011.

[54] J. A. Carpio, E. Massoni, and A. M. Gago, Revisiting quantum decoherence for neutrino oscillations in matter with constant density, Phys. Rev. D 97, 115017 (2018). 
[55] A. M. Gago, E. M. Santos, W. J. C. Teves, and R. Zukanovich Funchal, Quantum dissipative effects and neutrinos: Current constraints and future perspectives, Phys. Rev. D 63, 073001 (2001).

[56] R. Brustein, D. Eichler, and S. Foffa, Probing the Planck scale with neutrino oscillations, Phys. Rev. D 65, 105006 (2002).

[57] I. Esteban, M. C. Gonzalez-Garcia, M. Maltoni, I. MartinezSoler, and J. Salvado, Updated constraints on non-standard interactions from global analysis of oscillation data, J. High Energy Phys. 08 (2018) 180.

[58] S. Weinberg, A Model of Leptons, Phys. Rev. Lett. 19, 1264 (1967).

[59] M. Chalmers, Interview: Steven Weinberg, CERN Courier 57, 31 (2017), https://cds.cern.ch/record/2289267/files/CERN\% 20Courier\%20Volume $\% 2057 \% 20$ Issue $\% 209 \% 20$ (November\% 202017).pdf. 